\title{
Evaluation System of Open Platform Cameras for Bio-Imaging ${ }^{\dagger}$
}

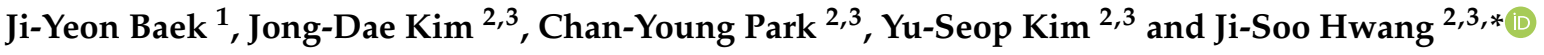 \\ 1 Help-Me Law Firm, Seoul 06158, Korea; qorwldus0310@naver.com \\ 2 School of Software, Hallym University, Chuncheon 24252, Korea; kimjd@hallym.ac.kr (J.-D.K.); \\ cypark@hallym.ac.kr (C.-Y.P.); yskim@hallym.ac.kr (Y.-S.K.) \\ 3 Bio-IT Research Center, Hallym University, Chuncheon 24252, Korea \\ * Correspondence: seattle@hallym.ac.kr; Tel.: +82-10-8930-5925 \\ + Presented at the 8th International Symposium on Sensor Science, 17-28 May 2021; Available online: \\ https://i3s2021dresden.sciforum.net/.
}

check for

updates

Citation: Baek, J.-Y.; Kim, J.-D.; Park, C.-Y.; Kim, Y.-S.; Hwang, J.-S. Evaluation System of Open Platform Cameras for Bio-Imaging. Eng. Proc. 2021, 6, 44. https://doi.org/10.3390/ I3S2021Dresden-10093

Academic Editors: Gianaurelio Cuniberti and Larysa Baraban

Published: 17 May 2021

Publisher's Note: MDPI stays neutral with regard to jurisdictional claims in published maps and institutional affiliations.

Copyright: (c) 2021 by the authors. Licensee MDPI, Basel, Switzerland. This article is an open access article distributed under the terms and conditions of the Creative Commons Attribution (CC BY) license (https:// creativecommons.org/licenses/by/ $4.0 /)$.

\begin{abstract}
With the development of smartphones, cameras based on ultra-small, high-definition, and open platforms have been mass-produced. In this paper, we outline how we built an emulation system to verify the bio-imaging performance of bulky and expensive high-performance cameras previously used in bio-imaging devices, and various smartphone cameras. Four types of camera were tested in the emulator, and the gel image analysis results were compared by selecting three cameras with more linear changes in slope, which matched the performance evaluation in the emulator.
\end{abstract}

Keywords: gel-document system; gel electrophoresis; bio-imaging; open platform camera

\section{Introduction}

The genetic information of living organisms is stored in DNA and RNA. It then replicates DNA through the process of cell division. Many studies of molecular biology techniques have been carried out, including confirmation of end-point amplification of samples in which specific DNA has been amplified by polymerase chain reaction (PCR), and genetics between objects. The gel-document system is an immediate device for taking images of the gel after electrophoresis for analysis of DNA fingerprinting used in research methods and forensics, and for analyzing base sequences [1,2].

There is a lot of commercial equipment using expensive CCD sensors and photographing equipment such as DSLRs. However, these sensors have a high price and a large focal length. Especially, in the case of DSLR, the focal length is too long and it is difficult to reduce the total size of the device.

With the development of mobile devices such as smartphones, it has become easy to obtain ultra-high-quality, low-cost open platform-based cameras. This camera is not only small in size, but also has a short focal length, which makes it possible to implement a small system at a low cost [3-5].

In this paper, we selected cameras with excellent linearity in both price range and size through an emulation test comparing the linearity of slope according to the brightness of the other three open platform cameras. To compare the performance with the DSLR frequently used in actual commercial machines, we compared the band volume of the agarose gel image after gel electrophoresis taken with Canon 1100D (DSLR), Sony IMX179, and AR0130. As a result, it was found that a DSLR has the best for maximum exposure, but the linearity of the DSLR and AR0130 is similar when the maximum exposure is matching with maximum exposure of AR0130. Therefore, it seems that the AR0130 can replace the bulky DSLR. This shows that even using an open platform camera can implement a small gel-document system at a sufficiently low cost. 


\section{Materials and Methods}

Figure 1 shows the composition of the proposed emulation system. The camera and the Arduino for lighting control are connected to the PC with a USB cable. The green LED was used as illumination, and the PWM was controlled through Arduino to change the brightness. The height of the LED and each camera in the emulator was fixed at a position where the aspect ratio was $80 \%$ when the test sheet was taken with each camera.

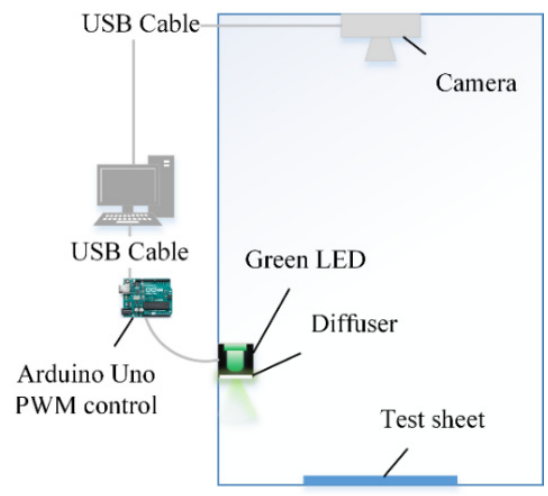

Figure 1. Gel document emulation system configuration.

In this paper, four types of camera were compared: Canon EOS1100D (DSLR), AR0130 web camera (AR0130), raspberry pi high-quality camera (PiCAM), and IMX179 web camera (IMX179). The first three cameras were installed $300 \mathrm{~mm}$ above the test sheet, $110 \mathrm{~mm}$ for the IMX179, and $75 \mathrm{~mm}$ for the Green LED. The shooting conditions for each camera were set based on the slope of the test sheet image at the LED brightness when PWM was 100 using the IMX179 camera installed in a gel-document system currently on sale. The emulator creates a dark room with black acrylic, and a light composed of a green LED and a diffuser can be adjusted with the PWM signal of Arduino Uno to configure the dark illumination.

First, in the test sheet image, a linear section with a relatively linear brightness change was found, and the equation of which order can be approximated was investigated.

The first to the sixth-order equation was used to find the minimum order at which the fitted error no longer decreases. The function obtained by fitting the calculated minimum order to the image taken in the brightest illumination for each camera was assumed as the reflection distribution function of the linear section of the test sheet image.

Next, when fitting with the same function while dimming the illumination, the function to be fitted was assumed to be the result of multiplying the function value by a constant less than 1.

To verify the proposed emulation system, a UV trans-illuminator was attached to the bottom and renovated to a gel-document system, which is one of the actual bio-imaging devices. Photography is conducted by attaching an emission filter to the DSLR used in the commercial system, and the IMX179, and the newly selected AR0130.

We added the nuclease-free water to the amplicon obtained by amplifying CT (Chlamydia trachomatis) DNA, diluted it to a concentration of $1,1 / 2,1 / 5,1 / 10,1 / 20$, and inserted it into agarose gel $(0.5 \times \mathrm{TBE})$. Then, electrophoresis was performed for 25 minutes. The gel was taken with the selected three types of camera, and the band volume for each concentration was observed.

Since the image sizes of the cameras used are all different, the gel images were matched equally using the OpenCV function, and the band images to be used for analysis were output. Since the brightness of the gel may decrease depending on the UV exposure time, two gels were used in the two camera experiments to alternately take pictures and compare.

In other words, in the comparative experiment between the DSLR and the AR0130, the first gel was taken in the order of DSLR $\rightarrow$ AR0130 $\rightarrow$ DSLR, and the second gel was 
photographed in the order of AR0130 $\rightarrow$ DSLR $\rightarrow$ AR0130, and then the band volumes of the images were compared and analyzed.

\section{Results}

Before comparing the efficiencies of each camera, we first used the fitting function to compare the brightness due to changes in the constant and PWM, and found that the results were in good agreement, which resulted in the brightness of the illumination. It was confirmed that the reflectance of each camera was constant according to the above.

The change in gradient was proportional to the brightness of all cameras, and the slope of AR0130 was the most linear, as shown in Figure 2. The more linear the brightness of illumination and the constant calculated using the function, the better the camera performance.

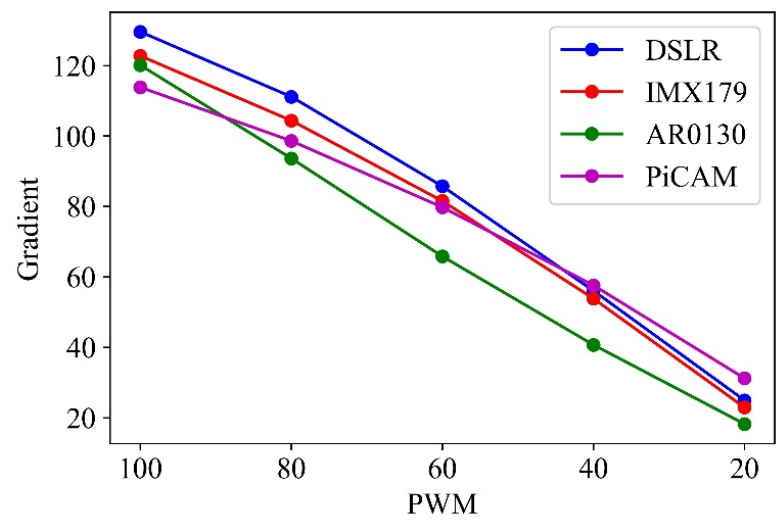

Figure 2. Gradient by the first PWM for each camera.

In this experiment, since the lighting was not uniform, it was difficult to draw conclusions only with a linear equation, so the analysis was performed by increasing the order. In the process of applying the fitting function, the linear function could not obtain the gradient of the straight line, and the photographed images with the brightest lighting were analyzed while increasing the order.

Also, the relationship between the PWM of each camera and the error of each order was analyzed. In the analysis results of all cameras, there was almost no difference in error in the third order or higher. In the analysis results of all cameras, there was almost no difference in error in the third order or higher, so it seems that the linearity of the gradient could be sufficiently compared using the result obtained from the third order equation.

Each band image was used for band volume analysis as shown in Figure 3. For each camera performance comparison, a coefficient of determination $\left(R^{2}\right)$ for the band volume was calculated, and a ranking comparison was performed on $R^{2}$. Then, the average $R^{2}$ value of the band volume of the first and third images and the $R^{2}$ value of the second image for each experiment, and their gradients, were compared. At this time, since the AR0130 and IMX179 were set to the maximum exposure, the brightness of the band volume was compared and analyzed together.

The reason for the shooting sequence in the order of DSLR $\rightarrow$ AR0130 $\rightarrow$ DSLR is that the third image will be darker than the first image, so there is also a need to check the change in the performance difference between the two cameras when they are analyzed in the same situation.

When comparing the band volume of each camera in four types of experiment, it was found that the DSLR was superior to the results of the ranking test of Experiment 1 and Experiment 2 concerning the R2 value. In Experiments 3 and 4, AR0130 was confirmed to have better performance than IMX179.

In comparing the average band volumes of the first and third images and that of the second image for each experiment, it was confirmed that the linearity of the DSLR and AR0130 was similar, and there was no significant difference when comparing the slope. 
This shows that the exposure value of the DSLR, which is the reference camera, is similarly matched to the maximum exposure of the AR0130. In the comparison experiment between AR0130 and IMX179, the linearity of AR0130 was better and, when comparing the slope, it was found that AR0130 better expresses the concentration in terms of brightness.

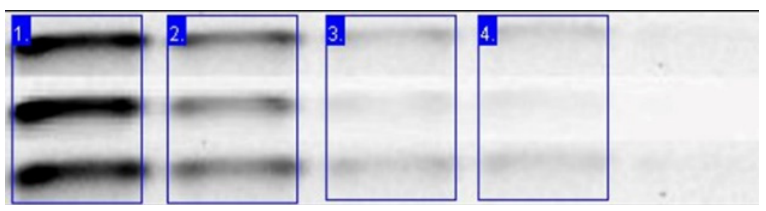

(a)

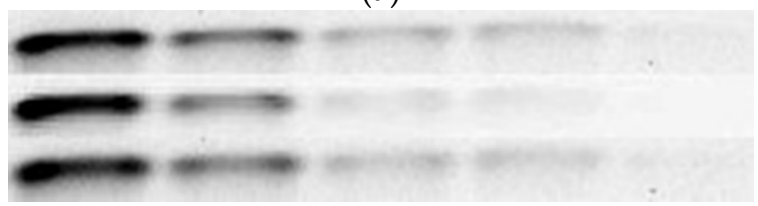

(b)

Figure 3. Gel image analysis: (a) band image (b) Exp \#1 band image.

\section{Conclusions and Discussions}

In this paper, we proposed an emulator that can evaluate whether an open platformbased smart phone camera is suitable for bio-imaging. Using the proposed emulation system, we compared the expensive DSLR cameras used in the gel-document system with three types of smartphone camera. In addition, when the gel after DNA electrophoresis was photographed and the band volumes of different reagent concentrations were compared, results consistent with the results of the emulator experiment were obtained.

The results of comparing the performance of open platform cameras in the proposed emulation system are likely to be sufficiently emulated if the conditions such as lighting and test sheets are changed not only in the gel-document system but also in other bio-imaging devices. However, since other devices have different photographing conditions, many experiments will have to be carried out in advance to apply the proposed system with an emulator.

Author Contributions: Conceptualization, J.-D.K.; methodology, J.-D.K.; software, J.-Y.B. and J.-D.K.; validation, J.-D.K. and J.-S.H.; formal analysis, J.-D.K. and J.-Y.B.; investigation, J.-S.H. and C.-Y.P.; resources, C.-Y.P. and Y.-S.K.; data curation, C.-Y.P. and Y.-S.K.; writing-original draft preparation, J.-Y.B.; writing-review and editing, J.-S.H.; visualization, J.-Y.B. and C.-Y.P.; supervision, J.-S.H.; project administration, J.-S.H.; funding acquisition, J.-S.H. All authors have read and agreed to the published version of the manuscript.

Funding: This research was funded by the National Research Foundation of Korea (NRF) grant funded by the Korea government (MOE) (NRF-2018R1D1A1B07051062) and the National Research Foundation of Korea (NRF) grant funded by the Korea government (MSIT) (NRF-2021R1A2C1011305).

Conflicts of Interest: The authors declare no conflict of interest.

\section{References}

1. Lee, P.Y.; Costumbrado, J.; Hsu, C.-Y.; Kim, Y.H. Agarose gel electrophoresis for the separation of DNA fragments. J. Vis. Exp. 2012, 62, e3923. [CrossRef]

2. Scott, T.; Dace, G.; Altschuler, M. Low-cost agarose gel documentation system. Bio. Tech. 1996, 21, 68-72. [CrossRef]

3. Goldmann, T.; Zyzik, A.; Loeschke, S.; Lindsay, W.; Vollmer, E. Cost-effective gel documentation using a web-cam. J. Biochem. Biophys. Methods 2001, 50, 91-95. [CrossRef]

4. Porch, T.G.; Erpelding, J.E. Low-cost conversion of the Polaroid MD-4 land camera to a digital gel documentation system. J. Biochem. Biophys. Methods 2006, 67, 1-5. [CrossRef]

5. Lee, D.J.; Kim, Y.R.; Kim, J.D.; Kim, Y.S.; Song, H.J.; Park, C.Y. Gel Documentation System Using an Open Platform Camera. Sens. Mater. 2019, 31, 287-292. [CrossRef] 